\title{
Comparison of survival outcomes between radical hysterectomy and definitive radiochemotherapy in stage IBI and IIAI cervical cancer
}

This article was published in the following Dove Press journal: Cancer Management and Research

\author{
San-Gang $\mathrm{Wu}^{1, *}$ \\ Wen-Wen Zhang',* \\ Zhen-Yu $\mathrm{He}^{2}$ \\ Jia-Yuan Sun ${ }^{2}$ \\ Yan Wang ${ }^{3}$ \\ Juan Zhou ${ }^{3}$
}

'Department of Radiation Oncology, Xiamen Cancer Hospital, The First Affiliated Hospital of Xiamen University, Xiamen, People's Republic of China; ${ }^{2}$ Department of Radiation Oncology, Sun Yat-sen University Cancer Center, State Key Laboratory of Oncology in South China,

Collaborative Innovation Center of Cancer Medicine, Guangzhou, People's Republic of China; ${ }^{3}$ Department of Obstetrics and Gynecology, The First Affiliated Hospital of Xiamen University, Xiamen, People's Republic of China

*These authors contributed equally to this work
Correspondence: Yan Wang; Juan Zhou Department of Obstetrics and Gynecology, The First Affiliated Hospital of Xiamen University, 55 Zhenhai Road, Xiamen 361003, People's Republic of China

Tel +86592 213 9707

Fax +86592 2137322

Email yanwangxmu@126.com;

juanzhoul2345@163.com
Introduction: There is an ongoing debate regarding the optimal local treatment modalities for stage IB1 and IIA1 cervical cancer. The aim of this study was to determine whether radical hysterectomy or definitive radiochemotherapy is superior in stage IB1 and IIA1 cervical squamous cell carcinoma (SCC).

Methods: From 1990 to 2010, a total of 3,769 patients with stage IB1 and IIA1 cervical SCC were included from the Surveillance, Epidemiology, and End Results database and were stratified according to whether they received radical hysterectomy or primary radiochemotherapy. Propensity score-matching (PSM) methods were used to balance patient baseline characteristics. Cancer-specific survival (CSS) and overall survival (OS) were compared between the two groups. Results: Of the 3,769 patients, 3,653 (96.9\%) and $116(3.1 \%)$ patients received radical hysterectomy and definitive radiochemotherapy, respectively. Radiochemotherapy was rarely used for definitive treatment prior to 2000. Before PSM, patients who were older, of black ethnicity, and with larger tumor size and stage IIA1 disease were more likely to receive definitive radiochemotherapy. A total of 116 pairs were completely matched using PSM. The local treatment modalities had no effect on CSS or OS in either unmatched or matched populations. In the matched population, the 8 -year CSS rates were $82.1 \%$ and $76.5 \%$ in surgery and radiochemotherapy groups, respectively $(p=0.382$ ). The 8 -year OS rates were $74.6 \%$ and $67.8 \%$ in surgery and radiochemotherapy groups, respectively $(p=0.205)$.

Conclusion: Our population-based study suggests that there is no clear local treatment of choice on survival outcomes between radical hysterectomy and definitive radiochemotherapy in patients with stage IB1 and IIA1 cervical SCC.

Keywords: cervical cancer, early stage, hysterectomy, radiotherapy, propensity score matching

\section{Introduction}

The incidence of cervical cancer (CC) has steadily declined over the last few decades coincident with the widespread, population-based screening. However, $\mathrm{CC}$ remains the third most common cancer in females and the fourth leading cause of cancer-related deaths in females worldwide, especially in low- and middle-income countries. ${ }^{1-3}$ For patients with locally advanced $\mathrm{CC}$, definitive radiochemotherapy is the standard treatment, ${ }^{4}$ while neoadjuvant chemotherapy + radical surgery was also a choice of treatment. $^{5-8}$ The standard treatment for stage IB1 and IIA1 CC is radical hysterectomy, whereas radiotherapy (RT) with or without chemotherapy is currently reserved as an optional treatment for nonoperative patients. ${ }^{9}$

Population-based studies have shown that most patients with stage IB-IIA CC underwent radical surgery rather than RT. ${ }^{10-12}$ One randomized study found that the 
survival outcomes between radical hysterectomy and primary RT were similar for stage IB-IIA cervical squamous cell carcinoma (SCC), whereas radical surgery was a better choice for patients with cervical adenocarcinoma. ${ }^{13}$ However, there is still ongoing debate regarding the optimal local treatment modalities for early-stage CC. Two previous population-based studies indicated that surgical treatment was associated with significantly better survival outcomes compared with primary RT in stage IB-IIA CC. ${ }^{10,11}$ A recent study of the National Cancer Database indicated that the addition of chemotherapy to definitive RT in stage IB1 and IIA1 CC improved overall survival (OS). ${ }^{14}$ The purpose of our study was to determine whether radical hysterectomy or definitive radiochemotherapy was superior in women with stage IB1 and IIA1 CC.

\section{Materials and methods}

\section{Patient identification}

Female patients with International Federation of Gynecology and Obstetrics (FIGO) stage IB1 and IIA1 CC who were treated between 1990 and 2010 were identified from the Surveillance, Epidemiology, and End Results (SEER) database. SEER is a population-based cancer registry representing approximately $28 \%$ of the United States population that is maintained by the National Cancer Institute. ${ }^{15}$ Patients who met the following criteria were included: 1) pathologically diagnosed with primary stage IB1 and IIA1 cervical SCC; 2) received either radical hysterectomy or definitive radiochemotherapy as the initial treatment (the RT cohort only included patients who received external-beam RT in combination with implants or isotopes); and 3) had available data for race/ethnicity, tumor grade, tumor size, and nodal status. This study was approved by the ethics committees of the First Affiliated Hospital of Xiamen University.

\section{Statistical methods}

Propensity score matching (PSM) was used to reduce selection bias in this study. ${ }^{16}$ The propensity score was defined as the probability of being assigned to the radical hysterectomy or definitive radiochemotherapy groups, given the demographic and clinicopathological variables. Propensity scores were computed by logistic regression for each patient with an algorithm of 1:1 matching using the following variables: age, race/ethnicity, tumor grade, FIGO stage, tumor size, and nodal status. ${ }^{17}$ Frequency distributions between categorical variables were analyzed by the $\chi^{2}$ test or Fisher's exact test, and analysis of variance was used to compare differences among the continuous variables. Survival time was plotted using the Kaplan-Meier method and compared using the log-rank test.
The primary endpoints of this study were cancer-specific survival (CSS) and OS. CSS was calculated for patients who died with CC-related disease. OS was defined as the time from treatment initiation until last follow-up or death. Univariate and multivariate proportional hazards models were used to analyze the prognostic factors for CSS and OS. The potential prognostic factors included age ( $<50$ years vs $\geq 50$ years), race/ethnicity (white vs black vs other), tumor grade (well/ moderately differentiated vs poorly/undifferentiated), tumor size (continuous variables), FIGO stage (IB1 vs IIA1), nodal status (negative vs positive), and treatment modalities (hysterectomy vs radiochemotherapy). The potential prognostic factors were initially compared using univariate analysis, and all variables that were significant in the univariate analysis were entered into the multivariate Cox regression model. Twosided $p$-values $<0.05$ were considered statistically significant. All statistical analyses were performed using SPSS 22.0 (IBM Corp., Armonk, NY, USA).

\section{Results}

There were 3,769 patients with stage IB1 and IIA1 CC identified in this study, including 3,653 (96.9\%) and $116(3.1 \%)$ patients who received radical hysterectomy and definitive radiochemotherapy, respectively. Surgery was the most common treatment modality used in patients. The use of radiochemotherapy as a definitive treatment was rarely seen before 2000 , with only three patients receiving radiochemotherapy from 1990 to $2000(p<0.001)$; the frequency of surgery or radiochemotherapy use is shown in Figure 1. A total of 1,311 patients $(35.9 \%)$ received adjuvant RT after radical hysterectomy. Patients who were older, of black ethnicity, and with larger tumor size and stage IIA1 disease were more likely to receive definitive radiochemotherapy. A total of 116 pairs were completely matched using PSM. The characteristics of the unmatched and matched populations are listed in Table 1.

The median follow-up for the entire cohort was 103 months. The 5- and 10-year CSS rates were $91.8 \%$ and $89.4 \%$, respectively, and the 5- and 10-year OS rates were $87.7 \%$ and $81.0 \%$, respectively.

In the univariate analysis of the unmatched population, radical hysterectomy was associated with better CSS and OS. However, the treatment arm was not identified as an independent prognostic factor on multivariate analysis. Age, race/ ethnicity, tumor grade, tumor size, FIGO stage, and nodal status were the independent prognostic factors of CSS and OS on multivariate analysis (Tables 2 and 3 ).

In the matched populations, the local treatment modalities also had no effect on CSS (hazard ratio [HR]: $0.770,95 \%$ 


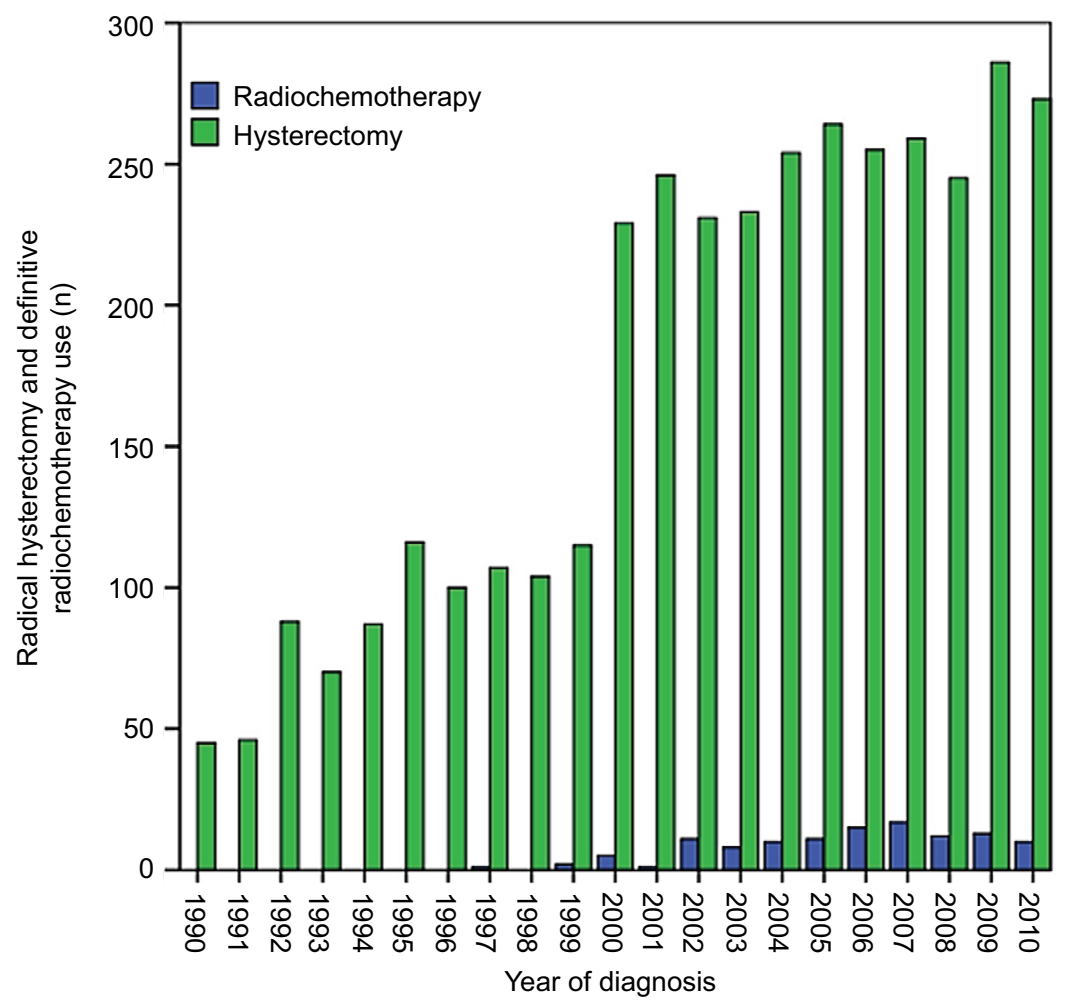

Figure I The frequency of radical hysterectomy and definitive radiochemotherapy use (1990-20I0).

Table I Patient characteristics of unmatched and matched populations

\begin{tabular}{|c|c|c|c|c|c|c|c|c|}
\hline \multirow[t]{2}{*}{ Variables } & \multicolumn{4}{|l|}{ Unmatched } & \multicolumn{4}{|l|}{ Matched } \\
\hline & Patients (N) & Surgery, n (\%) & RCT, n (\%) & p-value & Patients (N) & Surgery, n (\%) & RCT, n (\%) & $p$-value \\
\hline \multicolumn{9}{|l|}{ Age (years) } \\
\hline$<50$ & 2,564 & $2,504(68.5)$ & 60 (5।.7) & $<0.001$ & 116 & $56(48.3)$ & 60 (5I.7) & 0.599 \\
\hline$\geq 50$ & $\mathrm{I}, 205$ & $\mathrm{I}, \mathrm{I} 49$ (3I.5) & $56(48.3)$ & & 116 & 60 (5।.7) & $56(48.3)$ & \\
\hline \multicolumn{9}{|c|}{ Race/ethnicity } \\
\hline White & 2,926 & 2,839 (77.7) & $87(75.0)$ & 0.001 & 171 & $84(72.4)$ & $87(75.0)$ & 0.192 \\
\hline Black & 434 & $410(11.2)$ & $24(20.7)$ & & 44 & $20(17.2)$ & $24(20.7)$ & \\
\hline Others & 409 & 404 (II.I) & $5(4.3)$ & & 17 & $12(10.3)$ & $5(4.3)$ & \\
\hline \multicolumn{9}{|l|}{ Tumor grade } \\
\hline GI & 178 & $174(4.8)$ & $4(3.4)$ & 0.885 & 11 & $7(6.0)$ & $4(3.4)$ & 0.407 \\
\hline $\mathrm{G} 2$ & $\mathrm{I}, 722$ & $\mathrm{I}, 669$ (45.7) & $53(45.7)$ & & 97 & 44 (37.9) & $53(45.7)$ & \\
\hline G3 and 4 & 1,869 & $1,810(49.5)$ & $59(50.9)$ & & 124 & $65(56.0)$ & $59(50.9)$ & \\
\hline \multicolumn{9}{|c|}{ Tumor size (mm) } \\
\hline Mean & 22 & 22 & 31 & $<0.001$ & 31 & 31 & 31 & 0.815 \\
\hline \multicolumn{9}{|l|}{ FIGO stage } \\
\hline$|\mathrm{B}|$ & 3,520 & $3,428(93.8)$ & $92(79.3)$ & $<0.001$ & 183 & 91 (78.4) & $92(79.3)$ & 0.872 \\
\hline$|I A|$ & 249 & $225(6.2)$ & $24(20.7)$ & & 49 & $25(21.6)$ & $24(20.7)$ & \\
\hline \multicolumn{9}{|l|}{ Nodal stage } \\
\hline Negative & 3,099 & 3,009 (82.4) & $90(77.6)$ & 0.185 & 177 & $87(75.0)$ & $90(77.6)$ & 0.643 \\
\hline Positive & 670 & $644(17.6)$ & $26(22.4)$ & & 55 & $29(25.0)$ & $26(22.4)$ & \\
\hline
\end{tabular}

Abbreviations: GI, well differentiated; G2, moderately differentiated; G3, poorly differentiated; G4, undifferentiated; FIGO, International Federation of Gynecology and Obstetrics; RCT, radiochemotherapy.

confidence interval [CI]: $0.427-1.388, p=0.384$ ) or OS (HR: $0.732,95 \%$ CI: $0.450-1.189, p=0.207)$ on univariate analysis. The 8 -year CSS rates were $82.1 \%$ and $76.5 \%$ in surgery and radiochemotherapy groups, respectively (log-rank test, $p=0.382$; Figure $2 \mathrm{~A}$ ). The 8 -year OS rates were $74.6 \%$ and $67.8 \%$ in surgery and radiochemotherapy groups, respectively (log-rank test, $p=0.205$; Figure 2B). Race/ethnicity and tumor size were the independent prognostic factors for survival 
Table 2 Univariate prognostic analysis of unmatched and matched populations

\begin{tabular}{|c|c|c|c|c|c|c|c|c|}
\hline \multirow[t]{3}{*}{ Variables } & \multicolumn{4}{|l|}{ Unmatched } & \multicolumn{4}{|l|}{ Matched } \\
\hline & \multicolumn{2}{|l|}{ CSS } & \multicolumn{2}{|l|}{ os } & \multicolumn{2}{|l|}{ CSS } & \multicolumn{2}{|l|}{ OS } \\
\hline & HR (95\% CI) & $p$-value & HR (95\% Cl) & p-vlaue & HR (95\% CI) & $p$-value & HR (95\% Cl) & $p$-value \\
\hline \multicolumn{9}{|l|}{ Age (years) } \\
\hline$<50$ & 1 & & 1 & & 1 & & I & \\
\hline$\geq 50$ & $0.967(0.774-1.209)$ & 0.768 & $2.125(1.840-2.453)$ & $<0.001$ & $1.030(0.574-1.849)$ & 0.920 & 1.131 (0.702-I.822) & 0.613 \\
\hline \multicolumn{9}{|l|}{ Race/ethnicity } \\
\hline White & I & & 1 & & I & & I & \\
\hline Black & $1.273(0.947-|.7| 2)$ & 0.110 & $1.366(1.114-1.674)$ & 0.003 & $2.997(1.626-5.527)$ & $<0.001$ & $2.499(1.490-4.192)$ & $0.00 \mathrm{I}$ \\
\hline Others & 0.917 (0.648-I.297) & 0.624 & $0.948(0.748-\mid .202)$ & 0.658 & $0.718(0.170-3.028)$ & 0.652 & $0.852(0.305-2.379)$ & 0.760 \\
\hline \multicolumn{9}{|l|}{ Tumor grade } \\
\hline $\mathrm{GI}$ and 2 & I & & I & & I & & 1 & \\
\hline G3 and 4 & $1.640(1.329-2.024)$ & $<0.001$ & $1.347(1.165-1.556)$ & $<0.001$ & $2.295(1.204-4.373)$ & 0.012 & $1.969(0.184-3.273)$ & 0.009 \\
\hline Tumor size (mm) & $1.065(1.054-1.076)$ & $<0.001$ & $1.034(1.027-1.04 I)$ & $<0.001$ & 1.055 (1.015-1.097) & 0.007 & $1.033(1.004-1.062)$ & 0.023 \\
\hline \multicolumn{9}{|l|}{ FIGO stage } \\
\hline$|B|$ & I & & I & & I & & I & \\
\hline||$A \mid$ & $2.858(2.154-3.791)$ & $<0.001$ & $2.444(1.976-3.022)$ & $<0.001$ & $1.056(0.523-2.133)$ & 0.880 & $\mathrm{I} .440(0.848-2.447)$ & 0.177 \\
\hline \multicolumn{9}{|l|}{ Nodal stage } \\
\hline Negative & 1 & & 1 & & 1 & & 1 & \\
\hline Positive & $2.524(2.032-3.134)$ & $<0.001$ & $1.538(1.298-1.823)$ & $<0.001$ & $1.418(0.755-2.666)$ & 0.278 & 1.127 (0.658-1.932) & 0.664 \\
\hline \multicolumn{9}{|l|}{ Treatment } \\
\hline Radiochemotherapy & I & & I & & I & & I & \\
\hline Hysterectomy & $0.390(0.259-0.585)$ & $<0.001$ & $0.469(0.336-0.653)$ & $<0.001$ & $0.770(0.427-1.388)$ & 0.384 & $0.732(0.450-1.189)$ & 0.207 \\
\hline
\end{tabular}

Abbreviations: $\mathrm{Cl}$, confidence interval; CSS, cancer-specific survival; GI, well differentiated; G2, moderately differentiated; G3, poorly differentiated; G4, undifferentiated; FIGO, International Federation of Gynecology and Obstetrics; HR, hazard ratio; OS, overall survival.

Table 3 Multivariate prognostic analysis of unmatched and matched populations

\begin{tabular}{|c|c|c|c|c|c|c|c|c|}
\hline \multirow[t]{3}{*}{ Variables } & \multicolumn{4}{|l|}{ Unmatched } & \multicolumn{4}{|l|}{ Matched } \\
\hline & \multicolumn{2}{|l|}{ CSS } & \multicolumn{2}{|l|}{ OS } & \multicolumn{2}{|l|}{ CSS } & \multicolumn{2}{|l|}{ os } \\
\hline & HR (95\% Cl) & $p$-value & HR (95\% Cl) & p-value & HR (95\% Cl) & p-value & HR (95\% Cl) & $p$-value \\
\hline \multicolumn{9}{|l|}{ Age (years) } \\
\hline$<50$ & - & & 1 & & - & & - & \\
\hline$\geq 50$ & - & - & $2.084(1.800-2.413)$ & $<0.001$ & - & - & - & - \\
\hline \multicolumn{9}{|l|}{ Race/ethnicity } \\
\hline White & - & & I & & I & & I & \\
\hline Black & - & - & $1.317(1.075-1.615)$ & 0.008 & $2.755(\mathrm{I} .485-5.1 \mathrm{II})$ & 0.001 & $2.357(1.400-3.968)$ & 0.001 \\
\hline Others & - & - & $0.8 \mid 2(0.640-|.03|)$ & 0.088 & $0.716(0.169-3.034)$ & 0.650 & $0.834(0.298-2.337)$ & 0.730 \\
\hline \multicolumn{9}{|l|}{ Tumor grade } \\
\hline GI and 2 & I & & I & & I & & I & \\
\hline $\mathrm{G} 3$ and 4 & $1.382(1.118-1.708)$ & 0.003 & $1.219(1.054-1.41 \mathrm{I})$ & 0.008 & $1.793(0.925-3.475)$ & 0.084 & $1.682(0.999-2.834)$ & 0.050 \\
\hline Tumor size $(\mathrm{mm})$ & $1.053(1.042-1.065)$ & $<0.001$ & $1.028(1.020-1.035)$ & $<0.001$ & $1.046(1.006-1.087)$ & 0.023 & $1.026(0.998-1.055)$ & 0.073 \\
\hline \multicolumn{9}{|l|}{ FIGO stage } \\
\hline$|\mathrm{B}|$ & I & & I & & - & & - & \\
\hline$|I A|$ & $2.063(1.548-2.749)$ & $<0.001$ & $1.800(1.449-2.236)$ & $<0.001$ & - & - & - & - \\
\hline \multicolumn{9}{|l|}{ Nodal stage } \\
\hline Negative & I & & I & & - & & - & \\
\hline Positive & $1.864(1.494-2.325)$ & $<0.001$ & $1.375(1.155-1.636)$ & $<0.001$ & - & - & - & - \\
\hline \multicolumn{9}{|l|}{ Treatment } \\
\hline Radiochemotherapy & 1 & & 1 & & - & & - & \\
\hline Hysterectomy & $0.693(0.457-1.050)$ & 0.083 & $0.799(0.568-1.124)$ & 0.198 & - & - & - & - \\
\hline
\end{tabular}

Note: “-” indicates no data.

Abbreviations: Cl, confidence interval; CSS, cancer-specific survival; GI, well differentiated; G2, moderately differentiated; G3, poorly differentiated; G4, undifferentiated; FIGO, International Federation of Gynecology and Obstetrics; HR, hazard ratio; OS, overall survival. 
A

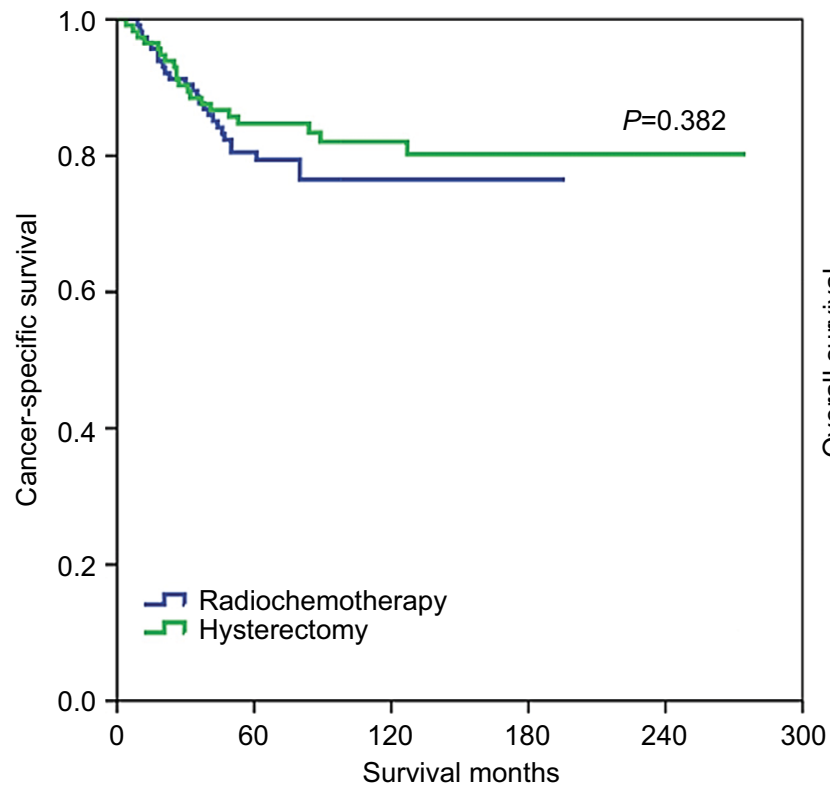

B

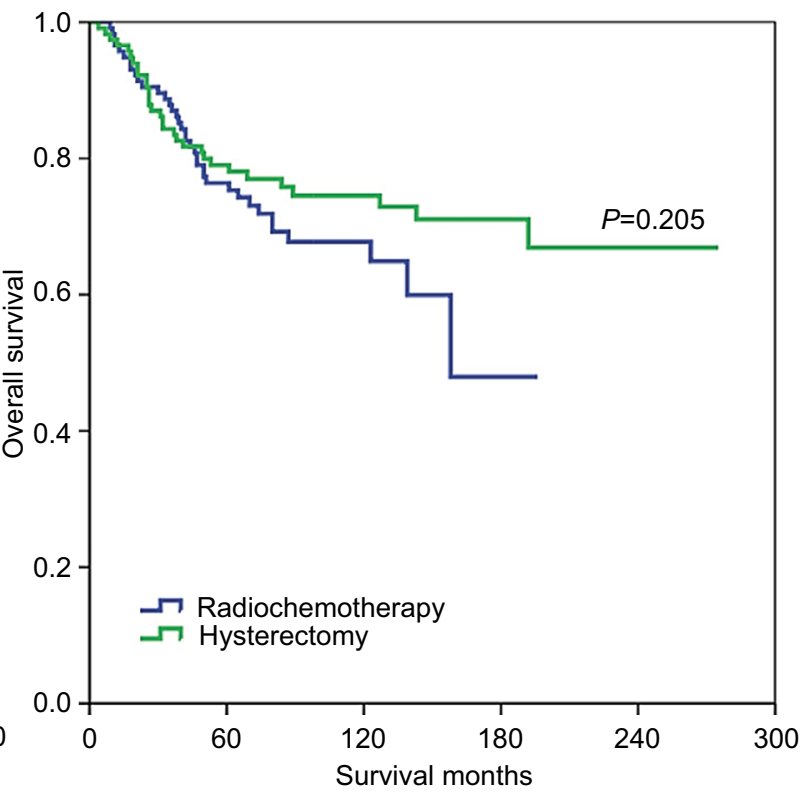

Figure 2 The CSS (A) and OS (B) between patients who underwent radical hysterectomy and definitive radiochemotherapy in the matched population. Abbreviations: CSS, cancer-specific survival; OS, overall survival.

outcome on multivariate analysis in matched populations (Tables 2 and 3).

\section{Discussion}

In this study, we analyzed the effects of different local treatment modalities on the survival of patients with stage IB1 and IIA1 cervical SCC using a population-based database. Our results found that the survival outcomes were similar between radical hysterectomy and definitive radiochemotherapy in both matched and unmatched populations.

The current National Comprehensive Cancer Network Guidelines recommend radical surgery or definitive RT with or without chemotherapy as alternative treatments for stage IB1 and IIA1 CC. ${ }^{9}$ However, surgical treatment is still the main local treatment used for early-stage CC. In our study, the vast majority of patients (96.9\%) received surgery-based treatment, and definitive radiochemotherapy was rarely used before 2000, which is related to the inclusion criteria in our study. Since 1999, five randomized trials have confirmed the clinical value of concurrent radiochemotherapy for bulky stage IB CC, high-risk early-stage CC after radical surgery, and locally advanced CC. ${ }^{18-22}$ In patients who received definitive RT, radiochemotherapy (66.7\%) was also the main treatment employed for stage IB1 and IIA1 CC in the recent National Cancer Database study and was associated with improved survival compared with definitive RT alone. ${ }^{14}$ The introduction of radiochemotherapy in the treatment of stage IB1 and IIA1 CC has gradually increased in recent years. However, radical surgery is still the most frequent treatment from 2000 to 2010.

To the best of our knowledge, there have been a total of five studies focusing on the survival differences between surgery and definitive RT/radiochemotherapy in patients with earlystage CC in the last two decades, ${ }^{10,11,23-25}$ and the optimal local treatment modality in these patients remains controversial. A randomized study by Landoni et $\mathrm{al}^{13}$ found equal survival rates between radical surgery and RT for stage IB-IIA cervical SCC. The 20-year OS rates associated with the two local treatment modalities were comparable in SCC subtype (72\% vs $77 \%, p=0.280) .{ }^{23}$ A retrospective study from Japan also found no statistically significant difference in survival for stage IA-IIB CC patients who received surgery $(\mathrm{n}=115)$ and those who received RT ( $\mathrm{n}=37 ; 79.9 \%$ vs $82.3 \%, p=0.8524) .{ }^{24}$ However, a study by Doll et a ${ }^{25}$ showed that radical hysterectomy $(n=169)$ significantly decreased the recurrence rate and improved survival with fewer disease complications compared with RT with or without chemotherapy $(n=29)$ in stage IB1 CC. In addition, two previous SEER studies found that surgical treatment was superior to RT for stage IB-IIA CC. . $^{10,11}$ In our study, we used PSM to reduce potential selection bias and found that definitive radiochemotherapy showed similar survival to radical hysterectomy for patients with stage IB1 and IIA1 cervical SCC in matched populations. However, radical hysterectomy group has approximately $20 \%-30 \%$ prognosis improvement, compared with radiochemotherapy group. Because of a small number of patients receiving 
definitive radiochemotherapy in our study, more prospective studies are needed to confirm our results.

Our results are similar to the randomized study by Landoni et al, ${ }^{13,23}$ but are contrary to the results of the two previous SEER studies. ${ }^{10,11}$ The relevant reasons may be as follows: 1) We only included patients with stage IB1 and IIA1 CC (tumor diameters $\leq 4 \mathrm{~cm}$ ), whereas patients with tumor diameters $>4 \mathrm{~cm}$ (stage IB2 and IIA2) were also included in the two previous SEER studies, in which larger tumor size was found to be an adverse prognostic factor. ${ }^{10,11}$ 2) Patients who received RT in our study also received chemotherapy, and several studies have shown that radiochemotherapy is associated with better survival than RT alone. ${ }^{14,18}$ The two previous SEER studies included patients from 1988 to 1995 and 1988 to 2005, respectively, such that most of the patients were not treated in the era of radiochemotherapy, which would have a potential impact on the results. In addition, the CSS and OS in our study were significantly higher than those in the two previous SEER studies. 3) We only included patients with the SCC subtype, whereas adenocarcinoma and adenosquamous carcinoma were also included in the previous SEER studies, and these histological subtypes have different RT sensitivity and chemosensitivity. ${ }^{26-30}$

Given the similar survival outcomes of patients undergoing radical hysterectomy and definitive radiochemotherapy, the pros and cons associated with each treatment modality play an important role in treatment selection. Surgery allows for the preservation of ovarian function and an accurate pathological assessment of nodal status. RT, on the other hand, is easy to deliver, which is particularly important in elderly patients, obese women, or patients with multiple medical comorbidities or contraindications to surgery. RT also avoids the laparotomy scar and anesthesia risks, and RT-induced iatrogenic mortality is rare. Furthermore, radical hysterectomy and definitive RT differ significantly in their accompanying morbidities and complications. Complications after RT occur later than surgery, although RT-related complications are usually permanent. The introduction of modern RT techniques such as intensity-modulated radiation therapy into clinical practice will yield much better results in terms of survival and morbidity, with significant reductions in chronic gastrointestinal, urinary, and vascular toxicities. ${ }^{31}$

Although $35.9 \%$ of patients received postoperative adjuvant RT in our study, approximately two-thirds of patients received adjuvant RT after radical hysterectomy in other studies of patients with IA-IIB stage CC. ${ }^{23,24}$ The incidence of complications in those studies was significantly different between the surgical arm and the RT arm (32\% vs $23 \%$, $p=0.006$ ), especially for leg edema. Leg edema occurred more often (11.1\%) in patients who received adjuvant RT after surgery compared with patients who underwent radical hysterectomy with lymphadenectomy (none) or RT alone $(0.6 \%){ }^{23}$ Therefore, adjuvant RT may increase the complication rates and decrease the quality of life of patients. Given the disparity in treatment-related morbidity, accurate preoperative assessment of the patients is more important, which could become a useful reference for treatment selection.

\section{Limitations}

We admit that our study has several limitations. First, although we used PSM to reduce potential selection bias, the SEER database lacks data on some pathologic variables that are important for $\mathrm{CC}$, including lymphovascular space invasion and depth of tumor invasion. Second, we included a limited number of patients receiving efinitive radiochemotherapy. In addition, the sequencing of RT and chemotherapy, the chemotherapy regimen, and the RT techniques and dosages are not recorded in the current SEER database. Moreover, we were unable to obtain the medical comorbidities present in patients before local treatment, which may impact treatment selection. Finally, the patterns of disease recurrence and treatment-related complications are also not available from the SEER database.

\section{Conclusion}

This population-based study further confirms that there is no treatment of choice on survival for patients with stage IB1 and IIA1 cervical SCC. The optimal treatment modality for the individual patient should take into consideration clinical factors such as age, body mass index, comorbidities, and sexual function. A prospective randomized trial is warranted to compare the survival and treatment-related complications of these two treatment modalities.

\section{Acknowledgment}

This work was supported by grants from the Natural Science Foundation of Fujian Province (number 2015J01550) and the Foundation Medical Innovation Foundation of Fujian Province (number 2015-CXB-34).

\section{Disclosure}

The authors report no conflicts of interest in this work.

\section{References}

1. Jemal A, Bray F, Center MM, et al. Global cancer statistics. CA Cancer J Clin. 2011;61(2):69-90.

2. Torre LA, Siegel RL, Ward EM, et al. Global cancer incidence and mortality rates and trends - an update. Cancer Epidemiol Biomarkers Prev. 2016;25(1):16-27. 
3. Angioli R, Lopez S, Aloisi A, et al. Ten years of HPV vaccines: state of art and controversies. Crit Rev Oncol Hematol. 2016;102:65-72.

4. Angioli R, Luvero D, Aloisi A, et al. Adjuvant chemotherapy after primary treatments for cervical cancer: a critical point of view and review of the literature. Expert Rev Anticancer Ther. 2014;14(4):431-439.

5. Luvero D, Plotti F, Aloisi A, et al. Patients treated with neoadjuvant chemotherapy + radical surgery + adjuvant chemotherapy in locally advanced cervical cancer: long-term outcomes, survival and prognostic factors in a single-center 10-year follow-up. Med Oncol. 2016;33(10):110.

6. Angioli R, Plotti F, Aloisi A, et al. A randomized controlled trial comparing four versus six courses of adjuvant platinum-based chemotherapy in locally advanced cervical cancer patients previously treated with neo-adjuvant chemotherapy plus radical surgery. Gynecol Oncol. 2015;139(3):433-438.

7. Wu SG, Zhang WW, Sun JY, Li FY, He ZY, Zhou J. Multimodal treatment including hysterectomy improves survival in patients with locally advanced cervical cancer: a population-based, propensity score-matched analysis. Int J Surg. 2017;48:122-127.

8. Angioli R, Plotti F, Luvero D, et al. Feasibility and safety of carboplatin plus paclitaxel as neoadjuvant chemotherapy for locally advanced cervical cancer: a pilot study. Tumour Biol. 2014;35(3):2741-2746.

9. National Comprehensive Cancer Network, Cervical cancer. 2017. (version 1). Available from: https://www.nccn.org/professionals/physician_gls/pdf/cervical.pdf. Accessed June 12, 2017.

10. Brewster WR, Monk BJ, Ziogas A, et al. Intent-to-treat analysis of stage $\mathrm{Ib}$ and IIa cervical cancer in the United States: radiotherapy or surgery 1988-1995. Obstet Gynecol. 2001;97(2):248-254.

11. Bansal N, Herzog TJ, Shaw RE, et al. Primary therapy for early-stage cervical cancer: radical hysterectomy vs radiation. Am J Obstet Gynecol. 2009;201(5):485.e1-e9.

12. Nogueira-Rodrigues A, Ferreira CG, Bergmann A, et al. Comparison of adenocarcinoma (ACA) and squamous cell carcinoma (SCC) of the uterine cervix in a sub-optimally screened cohort: a population-based epidemiologic study of 51,842 women in Brazil. Gynecol Oncol. 2014;135(2):292-296.

13. Landoni F, Maneo A, Colombo A, et al. Randomised study of radical surgery versus radiotherapy for stage Ib-IIa cervical cancer. Lancet. 1997;350(9077):535-540.

14. Haque W, Verma V, Fakhreddine M, et al. Addition of chemotherapy to definitive radiotherapy for IB 1 and IIA1 cervical cancer: analysis of the National Cancer Data Base. Gynecol Oncol. 2017;144(1):28-33.

15. Surveillance, Epidemiology, and End Results (SEER) Program (www. seer.cancer.gov) SEER*Stat Database: Incidence - SEER 18 Regs Research Data + Hurricane Katrina Impacted Louisiana Cases, Nov 2016 Sub (1973-2014 varying) - Linked To County Attributes - Total U.S., 1969-2015 Counties, National Cancer Institute, DCCPS, Surveillance Research Program, Surveillance Systems Branch, released April 2017, based on the November 2016 submission. Available from: https:// seer.cancer.gov/data. Accessed May 25, 2017.

16. Rosenbaum PR, Rubin DB. Constructing a control group using multivariate matched sampling methods that incorporate the propensity score. Am Stat. 1985;39(1):33-38.
17. Austin PC. An introduction to propensity score methods for reducing the effects of confounding in observational studies. Multivariate Behav Res. 2011;46(3):399-424.

18. Keys HM, Bundy BN, Stehman FB, et al. Cisplatin, radiation, and adjuvant hysterectomy compared with radiation and adjuvant hysterectomy for bulky stage IB cervical carcinoma. $N$ Engl J Med. 1999;340(15):1154-1161.

19. Morris M, Eifel PJ, Lu J, et al. Pelvic radiation with concurrent chemotherapy compared with pelvic and para-aortic radiation for high-risk cervical cancer. $N$ Engl J Med. 1999;340(15):1137-1143.

20. Rose PG, Bundy BN, Watkins EB, et al. Concurrent cisplatin-based radiotherapy and chemotherapy for locally advanced cervical cancer. N Engl J Med. 1999;340(15):1144-1153.

21. Whitney CW, Sause W, Bundy BN, et al. Randomized comparison of fluorouracil plus cisplatin versus hydroxyurea as an adjunct to radiation therapy in stage IIB-IVA carcinoma of the cervix with negative paraaortic lymph nodes: a Gynecologic Oncology Group and Southwest Oncology Group study. J Clin Oncol. 1999;17(5):1339-1348.

22. Peters WA 3rd, Liu PY, Barrett RJ 2nd, et al. Concurrent chemotherapy and pelvic radiation therapy compared with pelvic radiation therapy alone as adjuvant therapy after radical surgery in high-risk early-stage cancer of the cervix. J Clin Oncol. 2000;18(8):1606-1613.

23. Landoni F, Colombo A, Milani R, et al. Randomized study between radical surgery and radiotherapy for the treatment of stage IB-IIA cervical cancer: 20-year update. J Gynecol Oncol. 2017;28(3):e34.

24. Yamashita H, Nakagawa K, Tago M, et al. Comparison between conventional surgery and radiotherapy for FIGO stage I-II cervical carcinoma: a retrospective Japanese study. Gynecol Oncol. 2005;97(3):834-839.

25. Doll K, Donnelly E, Helenowksi I, et al. Treatment of stage IB1 cervix cancer: comparison of radical hysterectomy and radiation. Gynecol Oncol. 2011;123(2):444.

26. Huang YT, Wang CC, Tsai CS, et al. Long-term outcome and prognostic factors for adenocarcinoma/adenosquamous carcinoma of cervix after definitive radiotherapy. Int J Radiat Oncol Biol Phys. 2011;80(2):429-436.

27. Rose PG, Java JJ, Whitney CW, et al. Locally advanced adenocarcinoma and adenosquamous carcinomas of the cervix compared to squamous cell carcinomas of the cervix in gynecologic oncology group trials of cisplatin-based chemoradiation. Gynecol Oncol. 2014;135(2):208-212.

28. Chen JL, Huang CY, Huang YS, et al. Differential clinical characteristics, treatment response and prognosis of locally advanced adenocarcinoma/adenosquamous carcinoma and squamous cell carcinoma of cervix treated with definitive radiotherapy. Acta Obstet Gynecol Scand. 2014;93(7):661-668.

29. Zhou J, Zhang WW, Wu SG, et al. The prognostic value of histologic subtype in node-positive early-stage cervical cancer after hysterectomy and adjuvant radiotherapy. Int $J$ Surg. 2017;44:1-6.

30. Zhou J, Wu SG, Sun JY, et al. Comparison of clinical outcomes of squamous cell carcinoma, adenocarcinoma, and adenosquamous carcinoma of the uterine cervix after definitive radiotherapy: a population-based analysis. J Cancer Res Clin Oncol. 2017;143(1):115-122.

31. Wagner A, Jhingran A, Gaffney D. Intensity modulated radiotherapy in gynecologic cancers: hope, hype or hyperbole? Gynecol Oncol. 2013; 130(1):229-236.
Cancer Management and Research

\section{Publish your work in this journal}

Cancer Management and Research is an international, peer-reviewed open access journal focusing on cancer research and the optimal use of preventative and integrated treatment interventions to achieve improved outcomes, enhanced survival and quality of life for the cancer patient. The manuscript management system is completely online and includes

\section{Dovepress}

a very quick and fair peer-review system, which is all easy to use. Visit http://www.dovepress.com/testimonials.php to read real quotes from published authors. 